\title{
Intraoperative Radiotherapy of Breast Cancer and Its Biological Effects
}

\author{
Igor Piotrowski ${ }^{a}$ b Katarzyna Kulcenty ${ }^{a, b} \quad$ Mateusz Wichtowski ${ }^{c} \quad$ Dawid Murawa $^{c}$ \\ Wiktoria Suchorska ${ }^{a}$, b
}

${ }^{a}$ Radiobiology Laboratory, Department of Medical Physics, Greater Poland Cancer Centre, Poznań, Poland;

${ }^{b}$ Department of Electroradiology, University of Medical Sciences, Poznań, Poland;

${ }^{\mathrm{C}}$ Oncological and General Surgery Department I, Greater Poland Cancer Centre, Poznań, Poland

\section{Keywords}

Breast cancer - Radiotherapy - Molecular biology . Intraoperative radiotherapy

\section{Summary}

Conservative breast cancer surgery followed by radiation therapy is the standard treatment for this type of cancer. Numerous studies demonstrate that $90 \%$ of local recurrences after traditional surgery occur in the same quadrant as the primary cancer. The published data suggest that the wound healing process after surgery alters the area surrounding the original tumor and the modified microenvironment is more favorable for the tumor to recur. The majority of metastases within scar initiated much research, and the consequences of these studies led to clinical trials aimed at assessing whether localized radiotherapy, such as intraoperative radiotherapy (IORT), would be more effective in inhibiting formation of local recurrence than the standard postoperative whole breast radiotherapy. IORT involves irradiation of diseased tissue directly during surgery. The rationale for this approach is the fact that the increase in the radiation dose increases local tumor control, which is the primary goal of radiation therapy. The biological basis of this process are still not thoroughly understood. Gaining new knowledge about the recurrence formation at the molecular level could serve as a starting point for further analysis and to create an opportunity to identify new targets of therapy, and possibly new therapeutic agents.

\section{Introduction}

For almost a century, radical mastectomy was the treatment of choice for breast cancer patients. At the end of the last century, mastectomy was replaced by breast-conserving surgery followed by external beam radiation therapy (EBRT) delivered in fractioned doses, which significantly reduced the risk of local recurrence [1]. Even though EBRT achieves good results without inducing a high risk of side effects, the duration of this treatment is a major downside. Many patients who cannot attend a radiation center for several weeks of radiotherapy, receive mastectomy instead. Intraoperative radiotherapy (IORT) administered in 1 dose during surgery could be a solution to the problem of radiotherapy duration. The idea of using partial breast irradiation instead of whole breast irradiation derives from the fact that a great majority of intra-breast tumor reoccurrences arise in the same quadrant of the breast in which the primary tumor appeared [1]. Results of several ongoing IORT clinical trials show that IORT might be a viable alternative to EBRT for a specific group of patients. The effects of radiation and surgical procedures on tumor bed are still largely unknown. Researchers point out that the wound healing process and inflammation induced by the surgical procedure might stimulate the growth of residual cancer cells after tumor excision [2]. Recent discoveries indicate that this stimulatory effect might be reduced by radiation [3]. Better understanding of interactions occurring between breast cancer cells and tumor environment after radiation therapy might open the way for finding new targets for breast cancer therapy.

\section{Intraoperative Radiation Therapy Trials}

Different concepts of IORT have been adapted into therapy. IORT can be utilized as a boost and followed by irradiation of the

\section{KARGER}

(c) 2017 S. Karger GmbH, Freiburg
Greater Poland Cancer Centre

Garbary 15 Street, Poznań, Poland

katarzyna.kulcenty@wco.pl 
whole breast, which has provided promising results in local recurrence control [4]. A different approach of IORT is the usage of insertion techniques like MammoSite [5]. A disadvantage of this system is inhomogeneous irradiation of tumor bed. The trials that yielded the most promising results are electron intraoperative radiotherapy (ELIOT) and targeted intraoperative radiotherapy (TARGIT) $[6,7]$.

\section{ELIOT}

ELIOT is an electron IORT technique developed at the European Institute of Oncology (Milan, Italy) [6]. In this trial electrons are administered to tumor bed in a single session by a linear accelerator: NOVAC 7 or Liac. The NOVAC 7 accelerator is able to deliver collimated electron beams at energies of 3, 5, 7 and $9 \mathrm{MeV}$ [8]. In the preliminary study by Veronesi et al. [9], doses of 10, 15, 17, 19 and 21 Gy were administered to patients. Using a linear-quadratic surviving fraction model, scientists estimated that a single dose of 20-22 Gy is equivalent to 60 Gy delivered in 30 fractions of $2 \mathrm{~Gy}$, which is an accepted method of treatment following breastconserving surgery.

In the ELIOT trial, 1,305 patients between 48 and 75 years of age with tumors up to $2.5 \mathrm{~cm}$ in diameter were randomized [6]. 654 patients were assigned to external radiotherapy and $651 \mathrm{pa}-$ tients were assigned to ELIOT. For this trial, ipsilateral breast tumor recurrences (IBTR) included recurrences at the site of surgery and new carcinomas appearing in the same quadrant of the breast. 35 cases $(4.4 \%)$ of IBTR were observed in the ELIOT group compared to 4 cases $(0.4 \%)$ in the EBRT group. Patients in the IORT group developed IBTR at much higher rates. Characteristics that correlated with the highest IBTR rate (above 10\%) were: tumor size $>2 \mathrm{~cm}$, the presence of 4 or more positive nodes, tumor poorly differentiated, estrogen receptor-negative tumor and triple negative tumor. Authors concluded that patients with a tumor size of $>2 \mathrm{~cm}, 4$ or more positive lymph nodes, poorly differentiated tumor or triple negative tumor could potentially benefit from additional whole breast irradiation after IORT, administrated as a boost of $10 \mathrm{~Gy}$ [9]. It is worth mentioning that patients with a luminal A-subtype tumor had results similar to those of EBRT patients [10].

The ELIOT group also showed a higher rate of true local relapse (21 cases, $2.5 \%$ ) at 5 years than patients in the EBTR group (4 cases, $0.4 \%$ ). Distant metastases and primary cancers at other sites occurred with similar frequency in both groups. Overall 5-year survival was similar between the ELIOT and EBRT groups (96.8\% and 96.9\%, respectively).

Side effects of the therapy with IORT were assessed in 876 patients (464 in the ELIOT group and 412 in EBRT group). There were no significant differences in occurrence of mammary fibrosis, mammary retraction, pain and burning between the 2 groups. The IORT group suffered less often from skin-related side effects like erythema, dryness, hyper-pigmentation and pruritus compared to the EBRT group. More cases of liponecrosis were seen in the ELIOT than in the EBRT group.

\section{TARGIT-A}

The TARGIT-A trial is a randomized IORT trial comparing the effects of IORT performed with an intrabeam device to EBRT [7]. The device used in TARGIT-A has a low-energy x-ray source providing an isotropic dose of radiation by accelerating electrons into a gold target at the end of a $10-\mathrm{cm}$ probe. The energy of the photons produced is $50 \mathrm{keV}$, and the depth-dose of the $\mathrm{x}$-rays thus falls off rapidly [11]. With 20-45-min radiation, the tumor bed receives dose of $20 \mathrm{~Gy}$ that attenuates to 5-7 Gy at a depth of $1 \mathrm{~cm}$ [12]. The surgical procedure starts with a wide local excision and axillary clearance. After insertion of the appropriate applicator into the breast cavity, the breast tissue at risk is brought to the applicator by inserting a purse-string suture and irradiated [13].

Of the 3,451 patients aged 45 years or older with early breast cancer enrolled, 1,721 (49.87\%) were randomized to TARGIT and $1,730(50.13 \%)$ to EBRT. 239 of 1,571 patients receiving TARGIT (15.2\%) required supplemental EBRT. Additional EBRT was recommended when final pathology report showed 1 of 3 features: a positive margin of $>1 \mathrm{~mm}$, an extensive in-situ component, or invasive lobular carcinoma. The primary outcome of the trial was local recurrence in the conserved breast [7].

Risks of local recurrences in the conserved breast and other recurrences for patients who received TARGIT were 3.3\% and $4.9 \%$, respectively, compared with $1.3 \%$ and $4.4 \%$ in EBRT group. There was no significant difference between breast cancer-related deaths in TARGIT (2.6\%) and EBRT (1.9\%), but the TARGIT group showed fewer non-breast cancer deaths (1.4\%) than EBRT group (3.5\%). Patients who received both TARGIT-A and EBRT had a 3 times lower chance of developing local recurrence $(0.9 \%)$ and showed increased breast cancer mortality $(8 \%)$ in comparison with patients who received only TARGIT-A [7]. Median follow-up for the whole cohort at the end of trial was 29 months.

Silverstein et al. [14] in a critical review of the trial deemed 29 months of follow-up as too short a time to observe breast recurrences. The authors of the TARGIT-A trial emphasize the importance of selection of patients, and point out that a pre-pathology approach and risk-adapted design are crucial to allow the best results in patients.

Even though both TARGIT-A and ELIOT trials have already shown great promise in breast cancer therapy, it is necessary to significantly extend the follow-up. The results suggest that with proper selection of patients, these techniques will yield results similar to those of EBRT, while greatly reducing therapy time for the patients' convenience.

\section{Biological Aspects of IORT}

Even though recent results of IORT application in breast tumor treatment show great promise, our knowledge of biological and molecular effects of this therapy on tumor cells and tumor environment is still limited. Recently, direct effects of ionizing radiation on gene expression of luminal A breast cancer cell line MCF7 have been described [15-17]. The cells irradiated with 9 Gy and 23 Gy 
(IORT as boost and exclusive treatment, respectively) showed a senescence phenotype increasing with dose. Irradiation also caused double-strand breaks in a dose-dependent manner. Irradiated MCF-7 cells also showed an increased expression of proteins involved in stress and survival responses, but no evidence of apoptotic pathway activation. Radiation has been proven to increase NF-kB binding activity, leading to changes in cell-cycle regulation and to apoptosis suppression [18], which was also confirmed in the irradiated MCF-7 cells. These results show how the response of breast cancer cells differs depending on the administered radiation dose.

Kim et al. [19] described molecular effects of a radiation dose of 6 Gy on an MCF-7 cell line. Irradiated cells showed morphological changes increasing with dose, indicating senescence. The senescent phenotype also induced retardation in cell proliferation. Recent reports suggest that 'accelerated senescence' might be a major factor in loss of reproductive capacity by solid tumors [20]. Changes in the expression profile after irradiation resulted in cell cycle arrest at $\mathrm{S}$ and G2/M phases. Suppression of mitotic activity was also reflected by the fact that exposure of breast cancer cells to irradiation resulted in down-regulation of expression of the genes responsible for microtubule structure and for spindle organization and biogenesis [19].

The direct effect of cell irradiation, especially the DNA damage, has already been thoroughly described $[21,22]$. In addition to the direct effect, ionizing radiation can also affect non-irradiated cells neighboring the irradiated cells. This effect is called 'bystander effect' and is mediated through cell-to-cell gap junctions and secreted cytokines and chemokines [23]. Several mechanisms for the 'bystander effect' have already been described. It seems that induction of oxidative DNA damage by inflammatory response is an important factor [24]. Although the changes in microenvironment can inhibit tumor growth, researchers point out that mediators of the 'bystander effect' can also induce tumor growth [25]. The radiation-mediated 'bystander effect' increases with dose, but reaches plateau at relatively low doses $[23,26]$. The effect of ionizing radiation on carcinoma cells growing in a mammary stromal microenvironment and on the microenvironment itself has been demonstrated. Researchers have investigated interactions in 3-dimensional (3D) co-culture between fibroblasts exposed to chronic lowdose radiation with non-irradiated breast cancer cells (MDA-MB-231) and with untransformed mammary epithelial cells MCF-10A. Results show that irradiated fibroblasts exhibited characteristics of replicative senescence. Irradiated fibroblasts also disrupted development of MCF-10A into ductal structures. In co-culture with MDA-MB-231 cells, senescence-like fibroblasts induced growth and invasion of breast carcinoma cells. It was concluded that irradiated fibroblasts secreted matrix-degrading metalloproteinases, which effect the invasive growth of breast cancer cells. The authors pointed out that accumulation of senescence-like fibroblasts in breast stroma might create environment that promotes breast cancer progression [27].

Local relapse is often pointed out as the most important risk factor in survival of breast cancer patients [28]. Most local recurrences occur within the same quadrant as the primary cancer, sug- gesting that cancer cells remaining in tumor bed after surgery might be responsible for that. The changes in the tumor microenvironment, such as inflammation and wound healing initiated by surgery, might promote growth of breast cancer cells [29]. A recent study conducted by Beletti et al. [3] indicated that IORT modifies properties of surgical wound fluids (WF). Breast cancer cells cultured with WF harvested from IORT-treated patients showed significantly reduced ability to grow in 3D culture, together with impaired motility and cell invasion in comparison to culture with surgical WF from patients who had not undergone IORT. The proteomic analysis of WF and WF from IORT-treated patients showed a decrease in molecules involved in tumor growth and motility in IORT WF. These results indicate the effect of radiation-induced changes in the microenvironment on breast cancer cells.

Surgical WF are also known to stimulate breast cancer cell growth, but the mechanisms of this stimulation remain largely unknown [29]. Recently, Segatto et al. [30] showed that stimulation of breast cancer cells with WF collected from patients after breast tumor excision induces the Signal Transducer and Activator of Transcription 3 (STAT3) pathway in those cells. STAT3 plays a role in the transcriptional regulation of genes responsible for cell proliferation, survival, self-renewal, differentiation and apoptosis [31] and has been proven to influence growth and progression of breast cancer cells $[32,33]$. These authors have shown that cell lines representing different pathological subtypes (basal MDAMB-231 and MDA-MB-468, luminal MCF-7, HER-2 positive BT474) displayed higher mammosphere-forming efficiency (MFE) and formed spheres bigger in size when stimulated with WF than after stimulation with epidermal growth factor (EGF), which is used as a standard sphere-forming agent. The authors confirmed that STAT3 mediates the effect of WF on mammosphere formation by showing that cells stimulated with WF in presence of STAT3 inhibitors had decreased MFE. Taken together with a fact that WF stimulation enriched the population of cancer cells with tumor-initiating cells, these results suggest that WF might be a potent factor inducing local recurrence[34]. Stimulation of breast cancer cells with WF in vitro also caused an increase in $\mathrm{CD} 44^{+} /$ $\mathrm{CD} 24^{- \text {low }}$ population, which is more tumorigenic and shows stem cell-like properties [35]. The effect of WF stimulation on the putative stem cell phenotype of breast cancer cells was also investigated by Zaleska et al. [36]. Although stimulation with both WF and WF from patients after IORT treatment induced ALDH1 activity in breast cancer cells (a marker connected with cancer-stem like phenotype $[35,37])$, WF collected from patients after surgery alone stimulated the ALDH1 activity more strongly than WF after IORT treatment. The effect of WF stimulation on expression of CD44 and CD24 varied significantly depending on the cell line. These results indicate that surgical procedure can influence the putative stem cell phenotype of breast cancer cells.

Another important factor in tumorigenesis is the activity of the 70-kDa ribosomal protein S6 kinase (p70S6K), a serine/threonine kinase involved in regulation of cell growth, survival and metabolism [38]. In primary breast cancer, amplification of the region $17 q 23$ containing the gene encoding p70S6K has been observed in 
$12.5 \%$ cases [39]. Robust activity of this protein has been connected to survival of residual cancer cells after surgery [34]. Experiments on breast cancer cell lines showed that stimulation with WF induced activity of p70S6K. In an in vivo model, breast cancer cells with impaired $\mathrm{p} 70 \mathrm{~S} 6 \mathrm{~K}$ formed tumors later and showed reduced invasion of surrounding tissue in comparison to control cells. Inhibition of p70S6K in breast cancer cells growing in vitro impaired their proliferation, survivability and growth even with WF stimulation [40]. The role of p70S6K in apoptosis escape, survival in hostile conditions and primary tumor growth makes it a viable target in breast cancer therapy. Interfering with its activity in cancer cells might be an important step in reducing locoregional recurrences.

\section{Summary}

Recent discoveries in the field of tumor microenvironment might help provide a better understanding of its importance in tumor growth and progression. High-dose radiation has been shown not only to directly induce damage on DNA but also to promote anti-tumor $\mathrm{T}$ cell immunity and expansion of activated
T cells [41]. It is important to note that changes in tumor microenvironment induced by irradiation may exert pro- and anti-tumor effects, since many cytokines and chemokines have unknown effects.

Application of IORT in breast cancer treatment has shown great promise not only by giving similar results to the widely used EBRT, but also by providing a treatment method that is less time and money consuming. This relatively new therapy is already used in many radiation centers around the world as an alternative to EBRT in the treatment of early breast carcinomas.

\section{Acknowledgement}

This work was supported by the National Science Centre (grant number: 2015/19/D/NZ5/02190).

\section{Disclosure Statement}

The authors indicate that there is no conflict of interest.

\section{References}

1 Veronesi U, Marubini E, Mariani L, et al.: Radiotherapy after breast-conserving surgery in small breast carcinoma: Long-term results of a randomized trial. Ann Oncol 2001;12:997-1003

2 Troester MA, Lee MH, Carter M, et al.: Activation of host wound responses in breast cancer microenvironment. Clin Cancer Res 2009;15:7020-7028.

3 Belletti B, Vaidya JS, D'Andrea S, et al.: Targeted intraoperative radiotherapy impairs the stimulation of breast cancer cell proliferation and invasion caused by surgical wounding. Clin Cancer Res 2008;14:1325-1332.

4 Reitsamer R, Sedlmayer F, Kopp M, et al.: The Salzburg concept of intraoperative radiotherapy for breast cancer: results and considerations. Int J Cancer 2006; 118:2882-2887.

5 Thompson N, Cogen C, Forman D, Londerville S: MammoSite Radiation Therapy System. Clin J Oncol Nurs 2005;9:375-377.

6 Veronesi U, Orecchia R, Maisonneuve P, et al.: Intraoperative radiotherapy versus external radiotherapy for early breast cancer (ELIOT): A randomised controlled equivalence trial. Lancet Oncol 2013;14:12691277.

7 Vaidya JS, Wenz F, Bulsara M, et al.: Risk-adapted targeted intraoperative radiotherapy versus whole-breast radiotherapy for breast cancer: 5-year results for local control and overall survival from the TARGIT-A randomised trial. Lancet 2014;383:603-613.

8 Veronesi U, Gatti G, Luini A, et al.: Intraoperative radiation therapy for breast cancer: technical notes. Breast J 2003;9:106-112.

9 Veronesi U, Orecchia R, Luini A, et al.: A preliminary report of intraoperative radiotherapy (IORT) in limited-stage breast cancers that are conservatively treated. Eur J Cancer 2001;37:2178-2183.

10 Esserman LJ, Alvarado MD, Howe RJ, et al.: Application of a decision analytic framework for adoption of clinical trial results: Are the data regarding TARGIT-A IORT ready for prime time? Breast Cancer Res Treat 2014;144:371-378.
11 Kraus-Tiefenbacher U, Steil V, Bauer L, et al.: A novel mobile device for intraoperative radiotherapy (IORT). Onkologie 2003;26:596-598.

12 Vaidya JS, Joseph DJ, Tobias JS, et al.: Targeted intraoperative radiotherapy versus whole breast radiotherapy for breast cancer (TARGIT-A trial): An international, prospective, randomised, non-inferiority phase 3 trial. Lancet 2010;376:91-102.

13 Vaidya JS, Tobias JS, Baum M, et al.: TARGeted Intraoperative radiotherapy (TARGIT): An innovative approach to partial-breast irradiation. Semin Radiat Oncol 2005; 15:84-91.

14 Silverstein MJ, Fastner G, Maluta S, et al.: Intraoperative radiation therapy: A critical analysis of the ELIOT and TARGIT trials. Part 2 - TARGIT. Ann Surg Oncol 2014;21:3793-3799.

15 Bravata V, Minafra L, Russo G, et al.: High-dose ionizing radiation regulates gene expression changes in the MCF7 breast cancer cell line. Anticancer Res 2015;35: 2577-2591.

16 Subik K, Lee JF, Baxter L, et al.: The expression patterns of ER, PR, HER2, CK5/6, EGFR, Ki-67 and AR by immunohistochemical analysis in breast cancer cell lines. Breast Cancer (Auckl) 2010;4:35-41.

17 Yan W, Chen Y, Yao Y, et al.: Increased invasion and tumorigenicity capacity of CD44+/CD24- breast cancer MCF7 cells in vitro and in nude mice. Cancer Cell Int 2013;13:62.

18 Veeraraghavan J, Natarajan M, Aravindan S, et al.: Radiation-triggered tumor necrosis factor (TNF) alphaNFkappaB cross-signaling favors survival advantage in human neuroblastoma cells. J Biol Chem 2011;286: 21588-21600.

19 Kim BC, Han NK, Byun HO, et al.: Time-dependently expressed markers and the characterization for premature senescence induced by ionizing radiation in MCF7. Oncol Rep 2010;24:395-403.
20 Gewirtz DA, Holt SE, Elmore LW: Accelerated senescence: An emerging role in tumor cell response to chemotherapy and radiation. Biochem Pharmacol 2008;76:947-957.

21 Goodhead DT: Initial events in the cellular effects of ionizing radiations: Clustered damage in DNA. Int J Radiat Biol 1994;65:7-17.

22 Henner WD, Grunberg SM, Haseltine WA: Sites and structure of gamma radiation-induced DNA strand breaks. J Biol Chem 1982;257:11750-11754.

23 Mothersill C, Seymour CB: Radiation-induced bystander effects - implications for cancer. Nat Rev Cancer 2004;4:158-164.

24 Sprung CN, Ivashkevich A, Forrester HB, et al.: Oxidative DNA damage caused by inflammation may link to stress-induced non-targeted effects. Cancer Lett 2015 356:72-81.

25 Park B, Yee C, Lee KM: The effect of radiation on the immune response to cancers. Int J Mol Sci 2014;15: 927-943.

26 Lara PC, Lopez-Penalver JJ, Farias Vde A, et al.: Direct and bystander radiation effects: A biophysical model and clinical perspectives. Cancer Lett 2015;356:5-16.

27 Tsai KK, Chuang EY, Little JB, Yuan ZM: Cellular mechanisms for low-dose ionizing radiation-induced perturbation of the breast tissue microenvironment. Cancer Res 2005;65:6734-6744.

28 Huston TL, Simmons RM: Locally recurrent breast cancer after conservation therapy. Am J Surg 2005;189: 229-235.

29 Tagliabue E, Agresti R, Carcangiu ML, et al.: Role of HER2 in wound-induced breast carcinoma proliferation. Lancet 2003;362:527-533.

30 Segatto I, Berton S, Sonego M, et al.: Surgery-induced wound response promotes stem-like and tumor-initiating features of breast cancer cells, via STAT3 signaling. Oncotarget 2014;5:6267-6279. 
31 Bromberg J: Signal transducers and activators of transcription as regulators of growth, apoptosis and breast development. Breast Cancer Res 2000;2:86-90.

32 Marotta LL, Almendro V, Marusyk A, et al.: The JAK2 STAT3 signaling pathway is required for growth of CD44(+)CD24(-) stem cell-like breast cancer cells in human tumors. J Clin Invest 2011;121:2723-2735.

33 Chang Q, Bournazou E, Sansone P, et al.: The IL-6/ JAK/Stat 3 feed-forward loop drives tumorigenesis and metastasis. Neoplasia 2013;15:848-862.

34 Segatto I, Berton S, Sonego M, et al.: Inhibition of breast cancer local relapse by targeting p70S6 kinase activity. J Mol Cell Biol 2013;5:428-431.
35 Al-Hajj M, Wicha MS, Benito-Hernandez A, et al.: Prospective identification of tumorigenic breast cancer cells. Proc Natl Acad Sci USA 2003;100:3983-3988.

36 Zaleska K, Suchorska WM, Przybyła A, Murawa D: Effect of surgical wound fluids after intraoperative electron radiotherapy on the cancer stem cell phenotype in a panel of human breast cancer cell lines. Oncol Lett 2016;12:3707-3714.

37 Croker AK, Goodale D, Chu J, et al.: High aldehyde dehydrogenase and expression of cancer stem cell markers selects for breast cancer cells with enhanced malignant and metastatic ability. J Cell Mol Med 2009; 13:2236-2252.
38 Fenton TR, Gout IT: Functions and regulation of the $70 \mathrm{kDa}$ ribosomal S6 kinases. Int J Biochem Cell Biol 2011;43:47-59.

39 Monni O, Barlund M, Mousses S, et al.: Comprehensive copy number and gene expression profiling of the 17 q23 amplicon in human breast cancer. Proc Natl Acad Sci USA 2001;98:5711-5716.

40 Segatto I, Berton S, Sonego M, et al.: p70S6 kinase mediates breast cancer cell survival in response to surgical wound fluid stimulation. Mol Oncol 2014;8:766-780.

41 Kaur P, Asea A: Radiation-induced effects and the immune system in cancer. Front Oncol 2012;2:191. 